Ergod. Th. \& Dynam. Sys. (1986), 6, 311-319

Printed in Great Britain

\title{
Stochastic stability of hyperbolic attractors
}

\author{
LAI-SANG YOUNG \\ Department of Mathematics, Michigan State University, East Lansing, MI 48824, USA
}

(Received 25 April 1985 and revised 22 October 1985)

Abstract. We study the effects of small random errors on the asymptotic distribution of points in the basin of a hyperbolic attractor.

Leaving precise statements and generalities for later, we give an indication of the type of result in this paper. Let $U \subset \mathbb{R}^{n}$ be a neighbourhood of a compact attracting set, i.e. $f \bar{U} \subset U$ for some map $f$. Suppose that as we apply $f$ to any point $x \in U$, instead of obtaining the precise value $f x$, the possible outcomes are given by the uniform distribution on an $\varepsilon$-disk centred at $f x$. Now we start this process at some arbitrary point and let $\mu_{e}$ be the asymptotic distribution. Our result says that if

$$
\Lambda=\bigcap_{n \geq 0} f^{n} U
$$

is a hyperbolic attractor, then $\mu_{\varepsilon}$ tends to the so-called Bowen-Ruelle-Sinai measure on $\Lambda$ as $\varepsilon \rightarrow 0$.

Recall that, according to the theory of Bowen, Ruelle and Sinai ([B], [BR], [Si]), if $\Lambda \subset U$ is a hyperbolic or Axiom A attractor, then

$$
\frac{1}{n} \sum_{i=0}^{n-1} \delta_{f^{i} x}
$$

tends to the B-R-S measure for Lebesgue-a.e. $x \in U$. Our result then can be interpreted as a statement of stochastic stability for hyperbolic attractors, in the sense that the asymptotic distributions of points in these systems are not drastically altered by the cumulative effects of small random errors.

Results very similar to ours were first obtained by Y. Kifer [K1], [K2]. His technical hypotheses do not coincide with ours, but more importantly, our perspectives are quite different. We view this process as the random composition of maps nearby $f$ (see $[F]$ ), and our goal is to demonstrate that under the constraints of our hypotheses this type of stochastic stability is a direct consequence of the persistence of hyperbolic structures and their equilibrium state measures.

\section{Preliminaries and statement of result}

Let $f: M \hookrightarrow$ be a $C^{2}$ diffeomorphism of a finite dimensional Riemannian manifold. Let $U \subset M$ be an open subset with compact closure and suppose that $f \bar{U} \subset U$. All the processes we consider take place inside $\bar{U}$. We assume that the errors that may 
occur as we apply $f$ to $x$ depend entirely on the point $x$. This puts us into the context of a Markov chain with stationary transition probabilities.

For $x \in \bar{U}$, let $p(\cdot \mid x)$ be a probability on the Borel subsets of $\bar{U}$. We think of $p(A \mid x)$ as the probability of sending $x$ into the set $A$. Thus for instance the probability of starting at $x_{0}$ and being in $A_{1}, A_{2}, \ldots, A_{n}$ successively is

$$
\int_{A_{n-1}} \int_{A_{n-2}} \cdots \int_{A_{1}} p\left(A_{n} \mid x_{n-1}\right) p\left(d x_{n-1} \mid x_{n-2}\right) \cdots p\left(d x_{1} \mid x_{0}\right) .
$$

Definition (1.1). Given transition probabilities $p(\cdot \mid x)$, a Borel probability measure $\mu$ on $\bar{U}$ is said to be invariant if

$$
\mu(A)=\int p(A \mid x) d \mu(x)
$$

for every Borel set $A$.

Let $\mathcal{M}(\bar{U})$ denote the space of Borel probability measures on $\bar{U}$, always endowed with the weak topology. The dirac measure at $x$ is denoted by $\delta_{x}$.

Proposition (1.2). (1) If $x \mapsto p(\cdot \mid x)$ is continuous, then an invariant measure exists.

(2) If $p(\cdot \mid x)$ is absolutely continuous with respect to Lebesgue measure (written $p(\cdot \mid x) \ll$ Leb.) for every $x$, then so are the invariant measures.

(3) Suppose for $\varepsilon>0, \mu_{\varepsilon}$ is an invariant measure corresponding to transition probabilities $p_{\varepsilon}$, and $p_{\varepsilon}(\cdot \mid x) \rightarrow \delta_{f x}$ uniformly in $x$ as $\varepsilon \rightarrow 0$, then all limit points of $\mu_{\varepsilon}$ are f-invariant.

Proof. We prove (1), leaving the rest as a straightforward exercise for the reader. Define $\mathscr{L}: \mathcal{M}(\bar{U}) \circlearrowleft$ by

$$
\int \phi d\left(\mathscr{L}_{\mu}\right)=\iint \phi(y) p(d y \mid x) d \mu(x),
$$

for every continuous function $\phi: \bar{U} \rightarrow \mathbb{R}$. Suppose $\mu_{n} \rightarrow \mu$. Since $x \mapsto \int \phi(y) p(d y \mid x)$ is continuous, it follows that

$$
\int \phi d\left(\mathscr{L} \mu_{n}\right) \rightarrow \int \phi d(\mathscr{L} \mu),
$$

which verifies the continuity of $\mathscr{L}$. Now pick any $\mu_{0} \in M(\bar{U})$. Limit points of the sequence $(1 / n) \sum_{i=0}^{n-1} \mathscr{L}^{i} \mu_{0}$ are the fixed points of $\mathscr{L}$, which are easily seen to satisfy definition 1.1 .

We return briefly to the uniform diffusion example mentioned in the introduction. One way of describing it is as follows: Let $D_{\varepsilon}=\left\{\alpha \in \mathbb{R}^{n}:\|\alpha\| \leq \varepsilon\right\}$ and write $f_{\alpha}=f+\alpha$. Then

$$
p_{\varepsilon}(A \mid x)=\nu_{\varepsilon}\left\{\alpha \in D_{\varepsilon}: f_{\alpha} x \in A\right\},
$$

where $\nu_{\varepsilon}$ is normalized Lebesgue measure on $D_{\varepsilon}$. Keeping this example in mind we study the following class of Markov chains:

Let $\Omega$ be a set of maps from $\vec{U}$ into itself. We consider transition probabilities of the form

$$
p(A \mid x)=\nu\{g \in \Omega: g x \in A\},
$$


where $\nu$ is a probability on $\Omega$. It is easy to verify that as long as $\Omega \subset C^{0}(\bar{U})$, the space of continuous maps from $\bar{U}$ into itself endowed with the $C^{\mathbf{0}}$-topology, and $\nu$ is a Borel measure with compact support, then $x \mapsto p(\cdot \mid x)$ is continuous and hence this process has an invariant measure.

Next we turn to the conditions we wish to impose on $f: U \leftrightarrows$.

Definition (1.3). A compact $f$-invariant set $\Lambda \subset M$ is said to be uniformly hyperbolic or simply hyperbolic if there is a continuous splitting of the tangent bundle over $\Lambda$ into a direct sum of two invariant subbundles $E^{u} \oplus E^{s}$ and that there are numbers $c>0$ and $\lambda>1$ s.t. for all $x \in \Lambda$ and $n \geq 0$,

$$
\begin{aligned}
& v \in E^{s}(x) \Rightarrow\left\|D f_{x}^{n} v\right\| \leq c \lambda^{-n}\|v\|, \\
& v \in E^{u}(x) \Rightarrow\left\|D f_{x}^{-n} v\right\| \leq c \lambda^{-n}\|v\| .
\end{aligned}
$$

Via a standard change of metric we may - and will - assume that $c=1$.

Definition (1.4). An invariant set $\Lambda \subset M$ is called a hyperbolic attractor with basin $U$ if:

(1) $U$ is an open set s.t. $f \bar{U} \subset U$ and

$$
\bigcap_{n \geq 0} f^{n} U=\Lambda
$$

(2) $\Lambda$ is a uniformly hyperbolic set; and

(3) $f \mid \Lambda$ has a dense orbit.

By the spectral decomposition theorem [B] every hyperbolic attractor $\Lambda$ can be decomposed into finitely many components $\Lambda_{1}, \ldots, \Lambda_{k}$ with $f \Lambda_{1}=\Lambda_{2}, f \Lambda_{2}=$ $\Lambda_{3}, \ldots, f \Lambda_{k}=\Lambda_{1}$. For simplicity we shall consider $f^{k} \mid \Lambda_{1}$ and call it $f$.

Definition (1.5). Let $\Lambda \subset U$ be a hyperbolic attractor with basin $U$. The Bowen-RuelleSinai measure (written 'B-R-S measure' from here on) is the unique measure $\mu$ with the property that for Lebesgue-a.e. $x \in U$,

$$
\frac{1}{n} \sum_{i=0}^{n-1} \delta_{f^{i} x} \rightarrow \mu \text {. }
$$

For further information regarding the theory of hyperbolic attractors see [B] or [Sh].

We now state the main result of this paper. Let $L(\cdot)$ denote the Lipschitz constant of a map. For $f \in C^{2}(\bar{U})$ and $\alpha, \beta>0$, let

$$
\Omega_{\alpha, \beta}(f)=\left\{g \in C^{1}(\bar{U}):\|f-g\|_{C^{1}} \leq \alpha \text { and } L(D g) \leq \beta\right\} .
$$

We put the $C^{1}$ metric on $\Omega_{\alpha, \beta}(f)$ (which makes it compact).

TheOREM (1.6). Let $f$ be a $C^{2}$ diffeomorphism of $M$ and suppose that $\Lambda \subset M$ is a hyperbolic attractor with basin $U$. Let $\alpha>0$ be sufficiently small and let $\beta>L(D f)$. For each $\varepsilon>0$, let $\nu_{\varepsilon}$ be a Borel probability measure on $\Omega_{\alpha, \beta}(f)$ with $\nu_{\varepsilon} \rightarrow \delta_{f}$ as $\varepsilon \rightarrow 0$ and let $\mu_{\varepsilon}$ be an invariant measure for the process defined by

$$
p_{\varepsilon}(A \mid x)=\nu_{\varepsilon}\{g: g x \in A\} \text {. }
$$

If $p_{\varepsilon}(\cdot \mid x) \ll$ Leb. for every $\varepsilon>0$ and $x \in \bar{U}$, then $\mu_{\varepsilon}$ tends to the $\mathrm{B}-\mathrm{R}-\mathrm{S}$ measure as $\varepsilon \rightarrow 0$.

We thank Y. Kifer for a suggestion in the formulation of this theorem. 


\section{Skew product representation}

We shall represent the type of Markov chains under consideration as skew products. Part A of this section concerns the measure-theoretic aspects of this representation while part $B$ is about the topological-differential structure of the resulting object.

(A) This subsection has nothing to do with hyperbolic attractors. Let $X$ be a compact metric space and let $\Omega$ be a compact subset of $C^{0}(X)$. Let $\Omega^{Z}$ denote the bi-infinite product of $\Omega$ with itself and define $F: \Lambda^{\mathbf{Z}} \times X \subseteq$ by

$$
F(g, x)=\left(\sigma g, g_{0} x\right)
$$

where $g=\left(\cdots g_{-1} g_{0} g_{1} \cdots\right)$ and $\sigma: \Omega^{Z} \circlearrowleft$ is the shift transformation, i.e. $(\sigma g)_{n}=$ $(g)_{n+1}$. If $\nu$ is a measure on $\Omega$, let $\nu^{Z}$ denote the product measure of $\nu$ with itself on $\Omega^{\mathbf{Z}}$. Finally, let $p_{1}: \Omega^{\mathbf{Z}} \times X \rightarrow \Omega^{\mathbf{Z}}$ and $p_{2}: \Omega^{\mathbf{Z}} \times X \rightarrow X$ be projections onto the first and second factors respectively.

Proposition (2.1). Let $\nu$ be a Borel probability measure on $\Omega$ and suppose that $\mu$ is an invariant measure for the process defined by

$$
p(A \mid x)=\nu\{g: g x \in A\} .
$$

Then $F^{n}\left(\nu^{\mathbf{Z}} \times \mu\right)$ converges weakly to an $F$-invariant measure $\mu^{*}$ on $\Omega^{\mathbf{Z}} \times X$. Moreover, $p_{1} \mu^{*}=\nu^{Z}, p_{2} \mu^{*}=\mu$.

Here $F^{n}\left(\nu^{\mathbf{Z}} \times \mu\right)(E)$ is defined to be $\left(\nu^{\mathbf{Z}} \times \mu\right)\left(F^{-n} E\right)$.

Proof. First we observe that once weak convergence is extablished, $\mu^{*}$ is automatically $F$-invariant. Also, $p_{1} \mu^{*}=\nu^{\mathbf{Z}}$ is obvious. To prove weak convergence let $\mathscr{B}_{n}$ be the $\sigma$-algebra on $\Omega^{\mathbf{Z}} \times X$ generated by sets of the form $E=\tilde{A} \times B$ where $B \subset X$ and $A \subset \prod_{-n}^{\infty} \Omega$ are Borel sets and $\tilde{A}=\left(\prod_{-\infty}^{-(n+1)} \Omega\right) \times A$. We write $m_{n}=F^{n}\left(\nu^{\mathbf{Z}} \times \mu\right)$ and will show that $m_{n+1}\left|\mathscr{B}_{n}=m_{n}\right| \mathscr{B}_{n}$ for all $n \geq 0$ by verifying this for $E \in \mathscr{B}_{n}$.

Let $m_{n}(\cdot \mid g)$ denote the conditional probability of $m_{n}$ on the fibre above $g$. Then

$$
m_{n}(E \mid g)= \begin{cases}\mu\left(g_{-n}^{-1} \cdots g_{-1}^{-1} E\right) & \text { if } g \in \tilde{A}, \\ 0 & \text { otherwise }\end{cases}
$$

so that

$$
m_{n}(E)=\int_{A} \mu\left(g_{-n}^{-1} \cdots g_{-1}^{-1} E\right) d\left(\prod_{-n}^{\infty} \nu\right)
$$

Now

$$
\begin{aligned}
m_{n+1}(E) & =\int_{\Omega \times A} \mu\left(g_{-(n+1)}^{-1} \cdots g_{-1}^{-1} E\right) d\left(\prod_{-(n+1)}^{\infty} \nu\right) \\
& =\int_{A}\left\{\int_{\Omega} \mu\left(g^{-1}\left(g_{-n}^{-1} \cdots g_{-1}^{-1} E\right)\right) d \nu(g)\right\} d\left(\prod_{-n}^{\infty} \nu\right) .
\end{aligned}
$$

To see that the inner integral equals $\mu\left(g_{-n}^{-1} \cdots g_{-1}^{-1} E\right)$, observe that for $C \subset X$,

$$
\int_{\Omega} \mu\left(g^{-1} C\right) d \nu(g)=\int_{X} \nu\{g: g x \in C\} d \mu(x)=\mu C .
$$

That $p_{2} \mu^{*}=\mu$ follows easily. 
(B) We now return to the situation where $f$ : $U \subseteq$ contains a hyperbolic attractor. Let $\beta>L(D f)$ be fixed and for small $\alpha$ write $\Omega_{\alpha}=\Omega_{\alpha, \beta}(f)$ (see the end of $\S 1$ for definitions).

Consider the skew product $F: \Omega_{\alpha}^{Z} \times \bar{U} \circlearrowleft$ as in $\S 2(\mathrm{~A})$. For $g \in \Omega_{\alpha}^{Z}$, let $U_{g}$ denote the $U$-fibre over $g$ and write the fibre map as $F_{g}: U_{g} \rightarrow U_{\sigma g}$. Then $F$ is a homeomorphism of $\Omega_{\alpha}^{Z} \times \bar{U}$ onto a subset of itself. Each fibre map $F_{g}$ is $C^{1}$ with $L\left(D F_{g}\right) \leq \beta$ and $(g, x) \mapsto D F_{g}(x)$ is continuous. Let $\tilde{\Lambda}=\bigcap_{n \geq 0} F^{n}\left(\Omega_{\alpha}^{Z} \times U\right)$. Then $\tilde{\Lambda}$ is a compact $F$-invariant set, fibre-wise attracting.

By the 'tangent space' at $(g, x)$ we refer always to the tangent space to $U_{g}$ at $x$. Likewise $T \tilde{\Lambda}=\bigcup_{(g, x) \in \tilde{\Lambda}} T_{(g, x)} U_{g}$.

Proposition (2.2). For sufficiently small $\alpha>0$, there is a continuous splitting of $T \tilde{\Lambda}$ into $\tilde{E}^{u} \oplus \tilde{E}^{s}$ and there exists $\tilde{\lambda}>1$ s.t. for all $(g, x) \in \tilde{\Lambda}$ and $n \geq 0$,

$$
\begin{aligned}
& v \in \tilde{E}^{u}(g, x) \Rightarrow\left\|D F_{g}(x) v\right\| \geq \tilde{\lambda}\|v\|, \\
& v \in \tilde{E}^{s}(g, x) \Rightarrow\left\|D F_{g}(x) v\right\| \leq \tilde{\lambda}^{-1}\|v\| .
\end{aligned}
$$

To prove this proposition we invoke the 'invariant cones argument', the standard argument used to prove the persistence of hyperbolic structures. We refer the reader to [Sh, chapters $7 \& 8$ ] for a detailed proof.

From here on we fix $\alpha$ small enough for the conclusion of proposition (2.2) to hold and write $\Omega=\Omega_{\alpha}$. For $(g, x) \in \tilde{\Lambda}$, we define

$$
\tilde{W}^{u}(g, x)=\left\{(g, y) \in \Omega^{Z} \times \bar{U}: d\left(F^{-n}(g, x), F^{-n}(g, y)\right) \rightarrow 0 \text { as } n \rightarrow \infty\right\} .
$$

The same proofs that work in the single transformation case show that for every $(g, x) \in \tilde{\Lambda}, \tilde{W}^{u}(g, x)$ is an immersed submanifold of $U_{g}$ and that $\tilde{W}^{u}(g, x) \subset \tilde{\Lambda}$. We shall call

$$
\tilde{W}^{u}(\tilde{\Lambda})=\bigcup_{(g, x) \in \tilde{\Lambda}} \tilde{W}^{u}(g, x)
$$

the 'unstable manifolds of $\tilde{\Lambda}$ '. These unstable manifolds form a continuous lamination of the compact set $\tilde{\Lambda}$.

We remark that the result of $\S 2(\mathrm{~A})$ is clearly not affected by our using the $C^{1}$ metric on $\Omega$.

\section{Measure absolutely continuous on unstable manifolds and proof of theorem}

Our proof of theorem (1.6) relies heavily on the Radon-Nikodym derivatives of certain measures along $\tilde{W}^{u}$-manifolds. First we give precise definitions.

Consider the hyperbolic attractor $f: \Lambda \varsigma$. If $L \subset W^{u}(x)$ is open in the leaf topology, let $d^{u}$ denote the induced Riemannian metric on $L$ and $m^{u}$ the corresponding Riemannian measure. Let $V$ be a Borel subset of $\Lambda$. Suppose $V$ is the disjoint union of a continuous family of open $W^{u}$-disks and $\mu$ is a Borel probability measure on $\Lambda$ with $\mu V>0$. For $x \in V$, let $V_{x}$ denote the $W^{u}$-disk in $V$ containing $x$ and let $\mu_{x}$ denote the conditional probability measure of $\mu$ on $V_{x}$

Definition (3.1.) (1) We say that $\mu$ has absolutely continuous conditional measures on $W^{u}$-manifolds if for every $V \subset \Lambda$ above, $\mu_{x} \ll m^{u}$ for $\mu$-a.e. $x$. 
(2) Suppose $\mu$ has absolutely continuous conditional measures on $W^{u}$. We say that the densities of $\mu$ on $W^{u}$ have bounded ratio if there exist $B, \delta>0$ s.t. for every $V$ above, $\rho=d \mu_{x} / d m^{u}$ satisfies

$$
\frac{1}{B} \leq \frac{\rho\left(z_{1}\right)}{\rho\left(z_{2}\right)} \leq B,
$$

for $m^{u}$-a.e. $z_{1}, z_{2} \in V_{x}$ with $d^{u}\left(z_{1}, z_{2}\right)<\delta$, $\mu$-a.e. $x$.

We omit the corresponding definitions for measures on $\tilde{\Lambda}$. The generalization is quite obvious.

Recall the following characterization of B-R-S measures:

THEOREM ([B], [Si]). The B-R-S measure is the unique f-invariant Borel probability measure on $\Lambda$ with absolutely continuous conditional measures on $W^{u}$.

Recall also that the B-R-S measure can be realized as the weak limit as $n \rightarrow \infty$ of $f^{n} m$, where $m=$ normalized Lebesgue measure on $U$.

Proof of theorem 1.6. Let $\left\{\nu_{\varepsilon}, \varepsilon>0\right\}$ be Borel probability measures on $\Omega$ converging weakly to $\delta_{f}$ as $\varepsilon \rightarrow 0$, and let $\left\{\mu_{\varepsilon}\right\}$ be invariant measures of the corresponding processes. In $\S 4$ we prove the following lemma.

LeMma (3.2). Let $\alpha>0$ be sufficiently small. Then for each $\varepsilon>0$, there is a unique F-invariant Borel probability measure $m_{\varepsilon}$ on $\tilde{\Lambda}$ satisfying:

(1) $p_{1} m_{\varepsilon}=\nu_{\varepsilon}^{Z}$; and

(2) $m_{\varepsilon}$ has absolutely continuous conditional measures on $\tilde{W}^{u}$-manifolds.

Moreover, the densities of all the $m_{\varepsilon}$ on $\tilde{W}^{u}$ have bounded ratios with a common bound depending only on $F$.

Now it follows from the hypothesis on $\nu_{\varepsilon}$ and proposition 1.2 (2) that each $\mu_{\varepsilon} \ll$ Leb. on $U$. Let $\mu_{\varepsilon}^{*}=\lim _{n \rightarrow \infty} F^{n}\left(\nu_{\varepsilon}^{Z} \times \mu_{\varepsilon}\right)$, as guaranteed to exist by proposition 2.1 .

LeMma (3.3). $\mu_{\varepsilon}^{*}=m_{\varepsilon}$.

This lemma is also proved in $\S 4$. Let $m^{*}$ be a limit point of $m_{\varepsilon}$ as $\varepsilon \rightarrow 0$. The bounded ratio property of the densities of $m_{\varepsilon}$ on $\tilde{W}^{u}$ is passed on to $m^{*}$. Since $m^{*}$ is supported on the single fibre over $g$ with $g_{n}=f$ for all $n, p_{2} m^{*}$ is $f$-invariant and so it must be the B-R-S measure on $\Lambda$. Quoting proposition 2.1 again we see that

$$
\mu_{\varepsilon}=p_{2} m_{\varepsilon} \rightarrow p_{2} m^{*}
$$

which is the desired conclusion.

We remark that for our purposes it is not really necessary to prove lemma 3.2. One could show directly that $\mu_{\varepsilon}^{*}$ has absolutely continuous conditional measures on $\tilde{W}^{u}$-manifolds. We leave that as an exercise.

\section{Proofs of lemmas}

Before giving the proofs of lemmas 3.2 and 3.3 we establish the following preliminary result. 
LEMMA 4.1. Let $L$ be an open disk in $\tilde{W}^{u}(g, x)$ for some $(g, x) \in \tilde{\Lambda}$ and let $m_{L}$ be the normalized Riemannian measure on $L$. Then any limit point $\mu$ of

$$
\frac{1}{n} \sum_{i=0}^{n-1} F^{i} m_{L}
$$

has absolutely continuous conditional measures on $\tilde{W}^{u}$-manifolds. Moreover, the densities of $\mu$ on $\tilde{W}^{u}$ have bounded ratio with $B$ and $\delta$ depending only on $F$.

The proof of this is almost identical to the corresponding one for a single hyperbolic attractor. We include a sketch for completeness.

Proof. For $\delta>0$, let $\tilde{W}_{\delta}^{u}(g, x)=\left\{(g, y) \in \tilde{W}^{u}(g, x): \tilde{d}^{u}(x, y) \leq \delta\right\}$ where $\tilde{d}^{u}$ and $\tilde{m}^{u}$ are analogous to $d^{u}$ and $m^{u}$ in $\Omega^{\mathbf{Z}} \times \bar{U}$. First there exist $A, \delta>0$ s.t. for every $(g, x) \in \tilde{\Lambda}$, if $z_{1}, z_{2} \in \tilde{W}_{\delta}^{u}(g, x)$ then

$$
\left|J\left(D F_{g} \mid \tilde{E}^{u}\right) z_{1}-J\left(D F_{g} \mid \tilde{E}^{u}\right) z_{2}\right| \leq A \tilde{d}^{u}\left(z_{1}, z_{2}\right) .
$$

This follows from the uniform Lipschitzness of the $\tilde{E}^{u}$-bundle along $\tilde{W}^{u}$-manifolds. (See e.g. $\$ 6.1$ of [HP]. A uniform bound on $L(D g)$ for all $g \in \Omega$ is needed here.) From this and the fact that $\tilde{d}^{u}\left(F^{-n} z_{1}, F^{-n} z_{2}\right) \leq \tilde{\lambda}^{-n} \tilde{d}^{u}\left(z_{1}, z_{2}\right)$, for all $z_{1}, z_{2} \in \tilde{W}^{u}(g, x)$ we obtain the following important estimate: there exists $B>0$ such that for all $(g, x) \in \tilde{\Lambda}$, and all $z_{1}, z_{2} \in \tilde{W}_{\delta}^{u}(g, x)$ and $n \geq 0$,

$$
\frac{1}{B} \leq \frac{\left|J\left(D g_{-n} \cdots \cdots \circ g_{-1} \mid \tilde{E}^{u}\right) z_{1}\right|}{\left|J\left(D g_{-n} \circ \cdots \circ g_{-1} \mid \tilde{E}^{u}\right) z_{2}\right|} \leq B .
$$

Suppose $\left(1 / n_{k}\right) \sum_{i=0}^{n_{k}-1} F^{i} m_{L} \rightarrow \mu$ for some subsequence $\left\{n_{k}\right\}$. We construct certain canonical neighbourhoods of $\tilde{\Lambda}$. Let $T$ be an embedded disk in $U$ having the same dimension as the bundle dimension of $\tilde{E}^{s}$ and with the property that for all $g \in \Omega^{\mathbf{Z}}$, $T$ is transverse to $\tilde{W}^{u}(\tilde{\Lambda}) \mid U_{\mathrm{g}}$. Let $\Sigma=\left(\Omega^{\mathbf{Z}} \times T\right) \cap \tilde{\Lambda}$. Then assuming $\delta$ is small enough, $V=\bigcup_{(g, x) \in \Sigma} \tilde{W}_{\delta}^{u}(g, x)$ is the disjoint union of a continuous family of $\tilde{W}^{u}$-disks. We may assume that $\mu V>0$ and $\mu(\partial V)=0$ (by shrinking $\Sigma$ or $\delta$ if necessary).

For each $n$, let $L_{n}=\left\{z \in L: F^{n} z \in \tilde{W}_{\delta}^{u}(g, x)\right.$ for some $(g, x) \in \Sigma$ but $\left.F^{n} L \not \supset \tilde{W}_{\delta}^{u}(g, x)\right\}$. Since $F$ expands distances uniformly along $\tilde{W}^{u}, m_{L}\left(L_{n}\right) \rightarrow 0$ as $n \rightarrow \infty$, so we can realize $\mu$ as $\lim _{n_{k} \rightarrow \infty}\left(1 / n_{k}\right) \sum F^{i}\left(m_{L} \mid\left(L-L_{i}\right)\right)$.

Suppose $F^{n}\left(L-L_{n}\right) \supset \tilde{W}_{\delta}^{u}(g, x),(g, x) \in \Sigma$ and

$$
\rho_{n}=\frac{d F^{n}\left(m_{L} \mid\left(L-L_{n}\right)\right)}{d \tilde{m}^{u}}
$$

on $\tilde{W}_{\delta}^{u}(g, x)$. Then

$$
\frac{1}{B} \leq \frac{\rho_{n}\left(z_{1}\right)}{\rho_{n}\left(z_{2}\right)} \leq B,
$$

for all $z_{1}, z_{2} \in \tilde{W}_{\delta}^{u}(g, x)$. These inequalities continue to hold for the conditional measures of the limit $\mu$.

Proof of lemma 3.2. Pick a $\nu_{\varepsilon}^{\mathbf{Z}}$-generic point $g$. Let $L \subset \tilde{W}^{u}(g, x)$ for some $x$, let $\mu$ be a measure constructed as in the last lemma and let $m_{\varepsilon}$ be an ergodic component of $\mu$. Since ergodic components of a measure contain entire $\tilde{W}^{u}$-leaves, $m_{\varepsilon}$ has absolutely continuous conditional measures on $\tilde{W}^{u}$ as well. Also, $\nu_{\varepsilon}^{\mathbf{Z}}$ is ergodic with respect to the shift transformation, so we may assume $p_{1} m_{\varepsilon}=\nu_{\varepsilon}^{Z}$. 
To prove uniqueness first consider $f: \Lambda \hookrightarrow$. Let $x, y \in \Lambda$ be nearby points. Define $[x, y]$ to be the unique point in $W_{\delta}^{u}(x) \cap W_{\delta}^{s}(y)$. Suppose $\delta$ is small enough that $N_{\delta}(x)=\left\{\left[z_{1}, z_{2}\right]: z_{1} \in W_{\delta}^{u}(x), z_{2} \in W_{\delta}^{s}(x)\right\}$ is well defined for every $x \in \Lambda$. Then there exist $x_{1}, \ldots, x_{k}$ s.t. $\bigcup_{i=1}^{k} N_{\delta / 4}\left(x_{i}\right) \supset \Lambda$. By the topological mixing property of $f$ ([B, p. 72]), there exists $N$ s.t. for all $i, j, 1 \leq i, j \leq k, f^{N} N_{\delta / 4}\left(x_{i}\right)$ 'crosses' $N_{\delta}\left(x_{j}\right)$, meaning that $f^{N} N_{\delta / 4}\left(x_{i}\right)$ contains an entire $W^{u}$-leaf in $N_{\delta}\left(x_{j}\right)$.

In the skew product $F: \Omega_{\alpha}^{Z} \times \bar{U} \subseteq,[(g, x),(g, y)]$ and $\tilde{N}_{\delta}(g, x)$ have the obvious meanings. If $\alpha$ is small enough, then the Hausdorff distance between $\Lambda$ and $U_{g} \cap \tilde{\Lambda}$ is small and $f^{N}$ is near $F^{N} \mid U_{g}$. So for every $g \in \Omega_{\alpha}^{Z}$, there exist $x_{1}, \ldots, x_{k} \in U_{g} \cap \tilde{\Lambda}$ and $y_{1}, \ldots, y_{k} \in U_{\sigma^{N} g} \cap \tilde{\Lambda}$ such that $\bigcup_{i=1}^{k} \tilde{N}_{\delta / 2}\left(g, x_{i}\right) \supset U_{g} \cap \tilde{\Lambda}, \bigcup_{i=1}^{k} \tilde{N}_{\delta / 2}\left(\sigma^{N} g, y_{i}\right) \supset$ $U_{\sigma^{N}} \cap \tilde{\Lambda}$, and $F^{N} \tilde{N}_{\delta / 2}\left(g, x_{i}\right)$ 'crosses' $\tilde{N}_{\delta / 2}\left(\sigma^{N} g, y_{j}\right)$ for every $i, j$.

Now let $m_{\varepsilon} \mid g$ be the conditional probability of $m_{\varepsilon}$ on $U_{g}$ for some $\nu_{\varepsilon}^{Z}$-generic $g$ and suppose $\left(m_{\varepsilon} \mid g\right) N_{\delta / 2}\left(g, x_{i}\right)>0$ for some $i$. Because the densities of $m_{\varepsilon}$ on $\tilde{W}^{u}$ are strictly positive wherever they are defined, there is a $\tilde{W}^{u}$-disk $L_{0}$ in $\tilde{N}_{\delta / 2}\left(g, x_{i}\right)$ with the property that $\tilde{m}^{u}$-a.e. $z \in L_{0}$ is $m_{\varepsilon}$-generic. The absolute continuity property of the $\tilde{W}^{s}$-foliation restricted to $U_{g}$ ([PS, p. 4]) then tells us that every $\tilde{W}^{u}$-disk $L$ in $\tilde{N}_{\delta / 2}\left(g, x_{i}\right)$ has the property that $\tilde{m}^{u}$-a.e. $z \in L$ is $m_{\varepsilon}$-generic. It follows from this and our choice of the $x_{i}^{\prime}$ 's and $y_{j}$ 's that $\tilde{m}^{u}$-a.e. $z$ in every $\tilde{W}^{u}$-leaf in $U_{\sigma^{N} g} \cap \tilde{\Lambda}$ is $m_{\varepsilon}$-generic, which proves the uniqueness of $m_{\varepsilon}$ with respect to (1) and (2) in the statement of lemma 3.1.

Proof of lemma 3.3. First there is an open set $\tilde{V} \subset \Omega^{\mathbb{Z}} \times U$ containing $\tilde{\Lambda}$ s.t. for every $g \in \Omega^{\mathrm{Z}}, U_{\mathrm{g}} \cap \tilde{V}$ is foliated by $\tilde{W}^{s}(\tilde{\Lambda})$. It follows from lemma 3.2 and the absolute continuity of this $\tilde{W}^{s}$-foliation on each $U_{g}$ that $\left(\nu_{\varepsilon}^{Z} \times\right.$ Leb. $)$-a.e. $(g, x)$ in $\tilde{V}$ is $m_{\varepsilon}$-generic. The same is true for $\left(\nu_{\varepsilon}^{\mathbf{Z}} \times\right.$ Leb. $)$-a.e. $(g, x)$ in $\Omega^{\mathbf{z}} \times U$ since $F^{n}\left(\Omega^{\mathbf{z}} \times U\right) \subset$ $\tilde{V}$ for all $n$ greater than some $n_{0}$. This together with the fact that $\mu_{\varepsilon} \ll$ Leb. implies that $\left(\nu_{\varepsilon}^{Z} \times \mu_{\varepsilon}\right)$-almost every point is $m_{\varepsilon}$-generic.

To complete the proof, let $\phi: \Lambda^{\mathbf{Z}} \times \bar{U} \rightarrow \mathbb{R}$ be a continuous function. Then

$$
\begin{aligned}
\int \phi d m_{\varepsilon} & =\int \lim _{n \rightarrow \infty} \frac{1}{n} \sum_{i=0}^{n-1} \phi \circ F^{i} d\left(\nu_{\varepsilon}^{\mathbf{Z}} \times \mu_{\varepsilon}\right) \\
& =\lim _{n \rightarrow \infty} \int \phi d\left(\frac{1}{n} \sum_{i=0}^{n-1} F^{i}\left(\nu_{\varepsilon}^{\mathbf{Z}} \times \mu_{\varepsilon}\right)\right) \\
& =\int \phi d \mu_{\varepsilon}^{*} .
\end{aligned}
$$

This research was supported in part by NSF.

\section{REFERENCES}

[B] R. Bowen. Equilibrium States and the Ergodic Theory of Anosov Diffeomorphisms. Lecture Notes in Math, Vol. 470, Springer-Verlag (1975).

[BR] R. Bowen \& D. Ruelle. The ergodic theory of Axiom A flows. Invent. Math. 29 (1975), 181-202.

[F] J. Franks. Time dependent stable diffeomorphisms. Invent. Math. 24 (1974), 163-172.

[HP] M. Hirsch \& C. Pugh. Stable manifolds and hyperbolic sets. AMS Proc. Symp. Pure Math. Vol. 14, (1970), 133-164. 
[K1] Y. Kifer. On small random perturbations of some smooth dynamical systems. Math. USSR Izvestija 8 (1974) 1083-1107.

[K2] Y. Kifer. General random perturbations of hyperbolic and expanding transformations. Preprint.

[PS] C. Pugh \& M. Shub. Ergodicity of Anosov actions. Invent. Math. 15 (1972) 1-23.

[Sh] M. Shub. Stabilité globale des systèmes dynamiques. Astérisque vol. 56 (1978). (English translation by J. Christy to appear.)

[Si] Ya. G. Sinai. Markov partitions and C-diffeomorphisms. Func. Anal. and its Appl. 2 (1968), No. 1, 64-89. 